\title{
ENERGETIC ELECTRON ANISOTROPIES IN THE MAGNETOTAIL: IDENTIFICATION OF OPEN AND CLOSED FIELD LINES
}

\author{
D. N. Baker and E. C. Stone
}

California Institute of Technology, Pasadena, California, 91125

Abstract. Unidirectional anisotropies in the energetic electron fluxes $(E \geqslant 200 \mathrm{keV})$ have been observed in the earth's magnetotail with the Caltech Electron/Isotope Spectrometer on IMP-8. The anisotropies occur during periods of enhanced fluxes and provide essential information on the topology (open or closed) of the magnetotail field lines which are associated with recently identified acceleration regions.

\section{Introduction}

The plasma and energetic particle populations in the earth's magnetotail have been studied extensively during the last decade. In contrast to the observation of anisotropies in the low energy proton fluxes ( $E \leqslant 50 \mathrm{keV}$ ) which are related to the bulk plasma flow patterns in the magnetotail, the observation of anisotropies in the energetic electron fluxes $(E \geqslant 45 \mathrm{keV})$ may provide critical information on the large scale configuration of the magnetic fields associated with possible local acceleration processes.

Earlier studies of energetic electrons within the magnetotail have, for the most part, either assumed a lack of anisotropy or else have emphasized the observed isotropy of the fluxes, with only brief mention of any observed anisotropies (see e.g., Bame, et al., 1967). Haskel1 (1969) found particle streaming present in the magnetosheath but not inside the magnetosphere or magnetotail. Retzler and Simpson (1969) found no statistically significant anisotropy for electrons with $E \geq 375 \mathrm{keV}$ and suggested that isotropy might be the result of either diffusive propagation or stochastic behavior of the field lines in the magnetotai1. Singer and Bame (1970) studied a large set of Vela passes through the magnetotail at $\sim 18 R_{E}$ and their conclusions for electrons $E \geq 33 \mathrm{keV}$ were as follows: (1) Strong energetic electron streaming, sporadic in nature, is present only in the magnetosheath, never in the magnetotail; and (2) Strong electron spin-modulation is observed inside the magnetotail, but this is the second-order (double-lobed) variety and is commonily seen only near the magnetopause at $\sim 18 R_{E}$.

On the ather hand, previous studies of magnetotail particles have shown that large bursts of highly anisotropic protons $\mathrm{E} \geqslant 0.3 \mathrm{MeV}$ are present within the magnetotai1. Armstrong and Krimigis (1968) found strong apparent streaming for such protons with flow only in the tailward direction. Hence it was concluded that the magnetotail field lines were open, based upon these proton anisotropy measurements. Later Fenne11

(1970) studied a larger set of proton burst data

Copyright 1976 by the American Geophysical Union. using similar instrumentation to that used by Armstrong and Krimigis (1968). The protons were often found to be highly anisotropic, but streaming was found to be both earthward and tailward, which would be unexpected if proton anisotropies were associated only with open field lines. In both the Armstrong and Krimigis (1968) and Fennell (1970) studies, when energetic electrons accompanied the proton bursts, the electrons were found to be isotropic.

These general findings have been recently reemphasized by Sarris et al. (1976) who have used IMP-7 data to show: (1) Unidirectional streaming of energetic protons and electrons $\left(E_{p}>0.29 \mathrm{MeV}\right.$, $\mathrm{E}_{\mathrm{e}}>0.22 \mathrm{MeV}$ ) is commonly observed ih the magnetosheath with flow in the tailward direction; (2) Strong proton streaming is observed within the magnetotail, both earthward and tailward in direction; and (3) Energetic electrons are essentially isotropic everywhere within the magnetotai1. Although not discussed by Sarris et a1., (1976), there is some indication in their data of occasional electron streaming with $j_{\max } / j_{\min } \sim 1.5$.

The new observations reported here are focused on a specific period of enhanced electron fluxes which is typical of only two periods (with a possible third example) observed in the magnetotail on IMP-8 during 1974. These periods have been identified by Frank et al. (1976) as encounters with regions of acceleration. We find significant unidirectional anisotropies in the energetic electrons ( $E 200 \mathrm{keV}$ ) during these periods.

\section{Observations}

The Caltech Electron/Isotope Spectrometer (EIS) on IMP-8 (Mewaldt et al., 1976) provides a high tempora1 resolution, low background instrument for measuring electrons $(E \neq 0.2 \mathrm{MeV})$ at $\sim 30 \mathrm{R}_{\mathrm{E}}$ geocentric distance. Angular distributions ${ }^{E}$ are measured in eight sun-fixed, $45^{\circ}$ sectors in the ecliptic plane. Each angular distribution is averaged over $\sim 20$ seconds, which corresponds to seven consecutive spacecraft rotations. Substantial fluxes of electrons with $E \geqslant 200 \mathrm{keV}$ are ordinarily measured in the plasma sheet, the boundary layer, and the magnetosheath on each pass through the magnetotai1. These energetic electron fluxes often have significant anisotropies associated with them. The 1974 data have been studied extensively and were the basis of preliminary reports (Baker, et al., 1975, 1976).

We report here on the period shown in Figure 1 which illustrates features observed in the pitch angle distributions of energetic electrons. The data were obtained on Aprit 18, 1974, near the center of the magnetotail at $\sim 33 R_{E}$. The flux of electrons $E \geqslant 200 \mathrm{keV}$ during this period, shown in the top panel of Figure 1 , is not unu- 


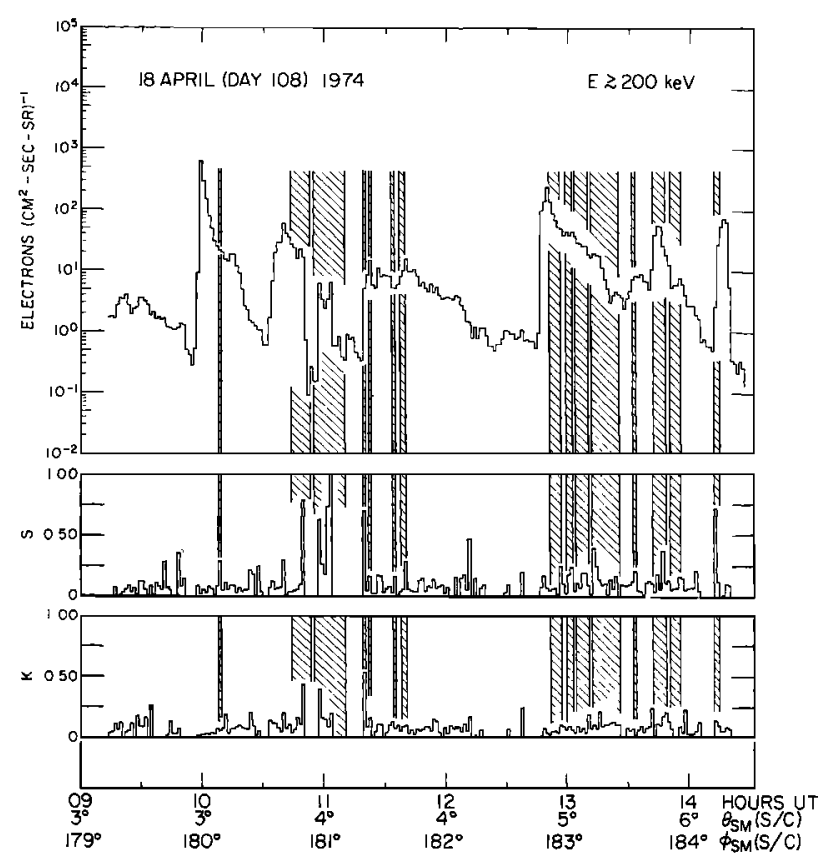

Figure 1. The absolute intensity of electrons $E \geqslant 200 \mathrm{keV}$ measured at $\sim 33 \mathrm{R}_{\mathrm{E}}$ geocentric distance on Apri1 18, 1974. The second and third panels from the top show the unidirectional, or streaming, amplitude and the second-order modulation amplitude, respectively. The spacecraft position in geocentric solar magnetospheric latitude and longitude is shown at the bottom of the figure. Regions where the average $Z$ component of the magnetic field is negative are shown by shaded bars.

sually large, but is quite variable. The magnetic field data from the Goddard Space Flight Center magnetometer also show extensive variability between 0900 and 1400 UT (R.P. Lepping, private communication, 1976). The field strength is often quite low ( $\$ 3 \times 10^{-5}$ gauss), the azimuthal direction is quite irregular, and, in contrast to the usual situation in the plasma sheet, the $Z$-component, $B_{Z}$, of the magnetic field is negative for substantial periods.

In Figure 1 we have shown the 82-second perjods in which the average $B_{7}$ is negative as vertical shaded bars. Several very brief and rather sporadic southward dips of the magnetic field are evident such as at 1010 UT, between 1115 and 1145 UT, and at $\sim 1410$ UT. Substantially longer and more durable southward excursions of $\mathrm{B}_{Z}$ are present at $\sim 1100$ and $\sim 1300$ UT.

The two lower panels of Figure 1 contain information about the observed anisotropy of the $E \geqslant 200 \mathrm{keV}$ electrons. We have fit the eight sector angular distributions using a simple Fourier expansion,

$$
C(\phi)=C_{0}\left[1+S \cos \left(\phi-\Delta_{1}\right)+K \cos 2\left(\phi-\Delta_{2}\right)\right] \text {. }
$$

Here $C$ is the number of counts observed in the azimuthal direction $\phi$, while $C_{0}$ is the spinaveraged number of counts. The expression in brackets represents the spin modulation with $S$ being the first-order (streaming) amplitude and $K$ being the second-order modulation amplitude. The parameters $\Delta_{1}$ and $\Delta_{2}$ represent the phase of the first-and second-order modulation, respec- tively. According to our definitions, $S$ (or $K$ ) lies in the range $0 \leq S \leq 1$ corresponding to the range of modulation from complete isotropy to extreme unidirectional (bidirectional) anisotropy. Fits are made according to Equation (1) for all sample perjods in which the spin-averaged flux exceeds $1\left(\mathrm{~cm}^{2}-\mathrm{sec}-\mathrm{s} r\right)^{-T}$. For fluxes below this limit, $\leqslant 3$ counts per sector are ordinarily present for the 20 second samples.

As is evident in Figure $1, S$ and $K$ are both small $(\leqslant 0.1)$ for substantial portions of the period shown, indicating near isotropy. This is commonly observed near the center of the magnetotail at $\sim 30 \mathrm{R}_{\mathrm{E}}$. However, during the course of several of the flux enhancements significant apparent modulation amplitudes are present, usually with $S$ larger than $K$. S values $\geqslant 0.3$ indicate streaming with $j_{\max } / j_{\min } \geqslant 2$. Near $\sim 1100$ UT, $S$ values are $\sim 0.8$, indicating detection of $\sim$ 10:1 unidirectional anisotropy. The figure shows that streaming amplitudes $S \geqslant 0.3$ during periods of high fluxes ( $>10 \mathrm{~cm}^{-2}-\mathrm{sec}^{-1}-\mathrm{sr}^{-1}$ ) are usually associated with regions of southward $\mathrm{B}_{Z}$. This is particularly clear near 1100 UT and after 1300 UT.

Figure 2 shows plots of five series of angular distributions. Such data were the basis of the computed parameters in portions of Figure 1. One angular distribution is assembled in $\sim 20$ seconds and is read out every $\sim 82$ seconds. Appropriate averages of magnetic field vectors are used for each $\sim 20$ second sample (hence employing finer time resolution than in Figure 1).

The first series from 0957 to 1007 UT was taken when $B_{Z}$ was consistently positive. From 0957 to 0958 the flux is seen to increase by a factor of $\sim 75$. A slower decline in flux is evident in the subsequent samples. The point to note, however, is that throughout this series, over a wide range of absolute intensities, the data are consistent with isotropy.

The second series of distributions from 1047 to 1103 UT was taken when $B_{7}$ switched from northto southward. There is a gapp in the sequence since fluxes dropped very low between 1050 and 1057 UT. The sample at 1047 when the magnetic field briefly had a northward component indicates approximate isotropy. All succeeding samples in the series are in regions of negative $B_{Z}$ and 10:1 streaming is detected. Note that to within the accuracy of the eight-sector sampling, the maxima and minima in the distributions lie along the local field line directions. Flow both parallel and antiparaliel to the field line is seen as the field azimuth rapidly changes, but the net flow is consistently tailward ( $i . e$. , the maximum intensity is detected at $\phi_{S E} \sim 0^{\circ}$ ).

In the third and fourth panels of Figure 2, $i t$ is seen that tailward streaming with $j_{\max } / j_{\min }$ in the range 2 to 10 is found mainly when $B_{Z}<0$.

A substantial flux enhancement later in the day (not shown in Figure 1) occurs when $B_{7}$ is solidly northward. In this sense the enhancement is more typical of the plasma sheet at $\sim 30 R_{E}$ and the fifth panel of Figure 2 shows angular distributions consistent with isotropy for most of this enhancement period. A distribution with a "cigar" character is seen at the time of peak intensities at 1632 UT.

The period from 0900 to 1400 UT described 


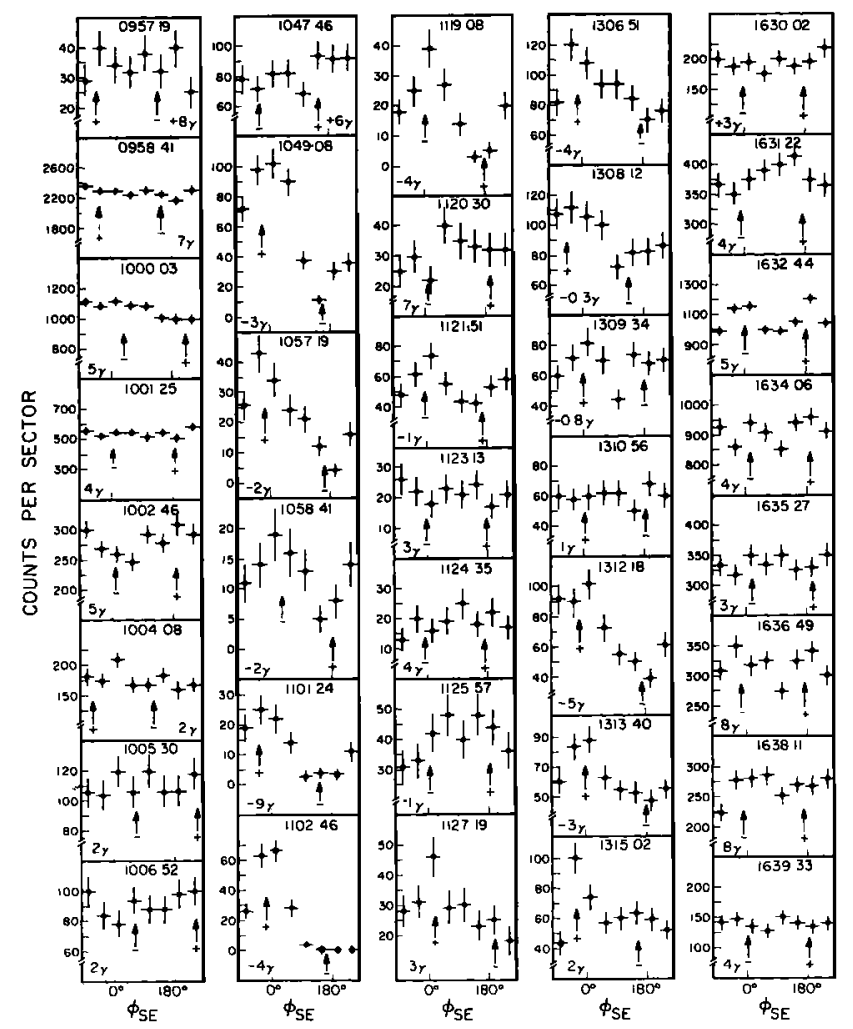

Figure 2. Sequences of angular distributions of electrons $E \geqslant 200 \mathrm{keV}$ for portions of the same day shown in Figure 1. The sample time (given as the nearest second UT at the beginning of the $\sim 20$ second sample period) and the measured value of $\mathrm{B}_{Z}$ (in gammas) for the sample period are shown for $\rightarrow$ each distribution. The azimuthal direction of $B$ is shown by vertical arrows for $\phi_{B}(+)$ and $\phi_{B}-180^{\circ}$ $(-)$. The number of counts per sector and the onesigma error bars are plotted versus solar ecliptic longitude.

here has been identified by Frank et al., (1976) as a localized region in which strong acceleration is occurring, probably due to magnetic field line merging. In regions where $\mathrm{BZ}$ is southward ( 1100 and $\sim 1300$ UT) tailward plasma jetting is reported by Frank et a1., with speeds often in excess of $1000 \mathrm{~km} \mathrm{sec-1.} \mathrm{In} \mathrm{regions} \mathrm{where}$ $B_{Z}$ is northward, the plasma flow is frequently strongly earthward and we find electrons $E \geqslant 200$ $\mathrm{keV}$ to show no strong streaming.

\section{DISCUSSION}

Energetic electrons have been frequently used as probes of magnetic field geometry near the earth. Electrons $E \geqslant 200 \mathrm{keV}$ seem well suited for this purpose since their velocity $(\geqslant 0.7 \mathrm{c})$ allows rapid traversal and bounce-stabilization of angular distributions on closed field lines, even for the very distended field lines at $30 R_{E}$ in the magnetotail plasma sheet. Unidirectional anisotropies identify open field lines through the absence of mirroring particles, while closed field lines may, by this argument, be identified by the presence of isotropic or other symmetric pitch angle distributions.

Even though strong energetic $(E>40 \mathrm{keV}$ ) electron streaming has not in the past been directly observed, such electrons, by virtue of their presence or absence, have been used extensively to indicate the large scale character of the plasma sheet and its magnetic topology. For example, Hones et al., (1974) have argued that their observation of relatively low fluxes of electrons ( $E \geq 43 \mathrm{keV}$ ) indicated that the Vela satellite at $\sim 18 R_{1}$ was on open field lines tailward of a magnetic neutral line. The electron flux was hypothesized to remain low because the electrons could escape relatively quickly along open field lines. These escaping electrons were found, however, to be not detectably spin-modulated. Hence it was suggested by Hones et al., that turbulence restrained the flow of electrons and randomized their pitch angle distributions.

Intensive investigation of the 1974 IMP-8 EIS data has shown that near-isotropy is, indeed, a common feature of electrons $E \geqslant 200 \mathrm{keV}$ deep within the magnetotail at $\sim 30 R_{E}$, but both moderately strong bidirectional ("cigar") and bimodal ("pancake") distributions are often found in association with intense plasma heating events (Baker et al., 1975, 1976). Strong unidirectional streaming of energetic electrons is a common feature of the magnetosheath and boundary layer (Singer and Bame, 1970; Sarris et a1., 1976; Baker et a1., 1976), but as summarized in the introduction, there have been no previous published reports of strong ( $\geqslant 2: 1)$ electron streaming within the magnetotail.

The almost complete absence of previous observations of streaming electrons causes one to consider other possible explanations of the kinds of data shown in Figures 1 and 2. Many examples of strong streaming occur as the electron flux is undergoing large variations and, thus, the possibility of time-aliasing has been considered. The effect that absolute intensity variations have can be modeled since the spacer,raft spin period and the sector sampling sequence are known. Flux changes by a factor of 50 in 20 seconds and having an exponential time variation, for example, give front-to-back sector ratios of $\sim 1.3$, corresponding to a smal1 streaming amplitude $(S<0.1)$.

Furthermore, for the IMP-8 EIS, an even-numbered sector $(0,2,4$, or 6$)$ is sampled first in a given sequence. Hence large, rapid intensity increases would produce the highest number of counts in the last sector sampled and the lowest number of counts in the first (even-numbered) sector sampled. The converse is true for rapid intensity decreases. Figure 2, in which the sectored data are always plotted left to right in the sector sequence 0 to 7 . shows relatively smooth variations from one sector to the next without the large discontinuities between adjacent sectors expected for time-aliasing.

other general indications of the reality of the observed anisotropies include: (1) The maximum and minimum fluxes track the azimuthal field direction; (2) Extreme intensity variations such as at 1000 UT (c.f. Figure 1) do not produce spuriously large anisotropies; (3) The streaming anisotropy effect is associated in some detail with $B_{7}$ variations. We conclude that the observed anisotropies are real and indicate an open field line topology.

The use of sub-MeV, non-thermal protons as field line tracers under conditions of large bulk plasma flow speeds $\left(1000-1500 \mathrm{~km} \mathrm{sec}^{-1}\right)$ is considerably complicated by the very large apparent proton anisotropies which result from the Compton-Getting effect. 
The large earthward anisotropy of protons $E \geqslant 0.3$ $\mathrm{MeV}$ is indicative of the importance of the ComptonGetting effect for protons. The energetic electrons discussed here are not subject to similarly large effects.

Our observations indicate that in rarely observed events, such as shown in Figure 1, the IMP-8 spacecraft encounters a region in the plasma sheet where strong energetic electron streaming is present. This tailward, field-aligned electron streaming is accompanied by tajlward plasma jetting in excess of $1000 \mathrm{~km} \mathrm{sec}^{-1}$ (Frank et al., 1976) and by southward $B_{Z}$. When plasma jetting is earthward and $\mathrm{B}_{7}$ is northward the $\mathrm{E} \geqslant 200 \mathrm{keV}$ electrons show no strong streaming. It seems probable that in such localized regions of particle acceleration, possible field line merging mechanisms and plasma sheet field line connection to the interplanetary medium can be most readily studied.

Acknowledgements. We thank L. A. Frank both for providing us with an extensive body of IMP-8 LEPEDA energy-time spectograms prior to publication and for informative discussions. We are also grateful to R. P. Lepping and N. F. Ness for providing us with the IMP-8 magnetometer data through J. H. King of the National Space Science Data Center. We are grateful to R. E. Vogt who has been closeiy involved in this investigation and to W. E. Althouse, G. J. Hurford, J. E. Lupton, and R. A. Mewaldt, who made significant contributions to various phases of this program. This work was supported in part by the National Aeronautics and Space Administration under contract NAS5-11066 and Grant NGR 05-002-160.

\section{References}

Armstrong, T. P. and S. M. Krimigis, Observations of Protons in the Magnetosphere and Magnetotail with Explorer 33, J. Geophys. Res., 73, 143, 1968.

Baker, D. N., E. C. Stone, R. E. Vogt, K. L. Ackerson, and L. A. Frank, Correlation of Electrons $E_{e}>0.2 \mathrm{MeV}$ with Low Energy Electrons ( $50 \mathrm{eV} \leq \mathrm{E} \leq 50 \mathrm{keV})$ in the Earth's Magnetotaii, Trans. Am. Geophys. Union, 56, $1046,1975$.
Baker, D. N., E. C. Stone, R. E. Vogt, L. A. Frank, and K.L. Ackerson, Energetic Electrons Observed in Conjunction with Magnetotail Fireball Events, Trans. Am. Geophys. Union, 57, 314, 1976.

Bame, S. J., J. R. Asbridge, H. E. Fel thauser, E. W. Hones, and I. B. Strong, Characteristics of the Plasma Sheet in the Earth's Magnetotail, J. Geophys. Res., 72, 113, 1967.

Fennel1, J.F., Observations of Proton Bursts in the Magnetotail with Explorer 35, J. Geophys. Res., 75, 7048, 1970.

Frank, L. A., K. L. Ackerson, and R. P. Lepping, On Hot Tenuous Plasmas, Fireballs, and Boundary Layers in the Earth's Magnetotail, submitted to J. Geophys. Res., 1976.

Haske11, G. P., Anisotropic Fluxes of Energetic Particles in the Outer Magnetosphere, J. Geophys. Res., 74, 1740, 1969.

Hones, E. W., Jr., A. T. Y. Lui, S. J. Bame, and S. Singer, Prolonged Tailward Flow of Plasma in the Thinned Plasma Sheet Observed at $r \approx 18 R_{E}$ During Substorms, J. Geophys. Res., 79, 1385, 1974.

Mewa7dt, R. A., E. C. Stone, S. B. Vidor, and R. E. Vogt, Isotopic and Elemental Composition of the Anomalous Low Energy Cosmic Ray Fluxes, Astrophys. J., 205, 931, 1976.

Retzler, J., and J. A. Simpson, Relativistic Electrons Confined Within the Neutral Sheet of the Geomagnetic Tail, J. Geophys. Res., 74, 2149, 1969 .

Sarris, E. T., S. M. Krimigis, and T. P. Armstrong, Observations of Magnetospheric Bursts of High Energy Protons and Electrons at $\sim 35 R_{E}$ with IMP-7, J. Geophys. Res., 81, 2341, 1976.

Singer, S., and S. J. Bame, Anisotropic Distributions of Energetic Electrons in the Earth's Magnetotail and Magnetosheath, in Particles and Fields in the Magnetosphere, edited by B. M. McCormac, p. 122, D. Reide1, Dordrecht, Netherlands, 1970.

(Received April 6, 1976;

revised Ju1y 6, 1976; accepted August 10, 1976.) 